\title{
Distância social dos profissionais de saúde em relação à dependência de substâncias psicoativas
}

\author{
Rhaisa Gontijo Soares \\ Universidade Federal de Juiz de Fora \\ Pollyanna Santos da Silveira \\ Universidade Federal de São Paulo \\ Leonardo Fernandes Martins \\ Henrique Pinto Gomide \\ Thais Medeiros Lopes \\ Telmo Mota Ronzani \\ Universidade Federal de Juiz de Fora
}

\begin{abstract}
Resumo
A atribuição de características indesejáveis a alguém pode conduzir à rejeição da pessoa rotulada, ocasionando o distanciamento social. O presente estudo teve como objetivos, avaliar as diferenças entre o desejo de distanciamento social dos profissionais de saúde da cidade de Juiz de Fora-MG, Brasil, em relação aos dependentes de álcool, maconha e cocaína, e possíveis relações entre o desejo de distanciamento social em cada um dos casos e variáveis sociodemográficas desses profissionais. Os resultados demonstraram que o distanciamento social foi maior para o dependente de cocaína, não havendo diferença significativa entre os escores das escalas para dependentes de álcool e maconha. Os julgamentos de distância social foram independentes de qualquer característica sociodemográfica da amostra, com exceção do aspecto "nível profissional" em relação ao dependente de álcool. O estudo do desejo de distância social dos profissionais de saúde pode contribuir para a implementação de estratégias de melhora dos serviços.
\end{abstract}

Palavras-chave: distância social; dependência a substâncias; estigmatização.

\begin{abstract}
Social distance among health professionals toward the substance dependence. The attribution of undesirable characteristics to a person may lead to rejection and social distance. This study aims to evaluate differences on desire for social distance related to dependents of marijuana, alcohol and cocaine, among health professionals from Juiz de Fora, Minas Gerais, Brazil. Relation between desire for social distance and the professionals socio-demographics characteristics was also availed. The results demonstrated greater social distance related to cocaine dependents and no significant difference between the scores for marijuana and alcohol dependents. Social distance judgements were not significantly correlated to any socio-demographics characteristics but "professional level", that correlated with "dependent of alcohol" scores. Studying social distances may contribute to the implementation of strategies that may lead to services improvement.
\end{abstract}

Keywords: social distance; substance dependence; stigmatization.

$\mathrm{O}$ sistema perceptivo humano, enquanto componente ativo do processo de seleção de estímulos ambientais, forma uma série de associações interligadas e coerentes sobre um objeto social percebido, a partir das primeiras impressões causadas e das experiências anteriores. Neste processo, a tendência a destacar elementos coerentes com a categorização inicial feita e eliminar aqueles que se opõem a ela, implica a atribuição de determinados rótulos, capazes de antecipar certos comportamentos de uma pessoa ou grupo, o que pode facilitar nossos relacionamentos ou distorcer nossas percepções
(Rodrigues, Assmar, \& Jablonski, 2005).

Segundo a Teoria da Rotulação, quando se aplica um rótulo a alguém, este rótulo identifica ou define de que tipo é essa pessoa (Link \& Phelan, 1999). Um rótulo é desviante quando é usado para designar a violação de uma norma social, e normal, quando é feita uma distinção que não envolve a violação de normas (Link \& Phelan, 1999). O processo de estigmatização ocorre quando há coexistência de rotulação, estereótipos, preconceito, perda de status e discriminação em uma situação de poder que permite que esses componentes interajam (Link \& Phelan, 2001). 
Muitos dos sintomas atribuídos a portadores de sofrimento mental resultam na aplicação de um rótulo desviante, por caracterizarem a violação de regras ou padrões sociais. A dificuldade de se separar a condição de saúde de uma pessoa do rótulo que essa carrega pode influenciar na disposição individual de alguém de engajar-se em atividades com tais pessoas (Link, Phelan, Bresnahan, Stueve, \& Pescosolido, 1999). Neste sentido, a atribuição de características indesejáveis a alguém pode conduzir à evitação e rejeição da pessoa rotulada, ocasionando o distanciamento social (Corrigan, Markowitz, Watson, Rowan, \& Kubiak, 2003; Link, Yang, Phelan, \& Collins, 2004).

O conceito de distância social, assim como a primeira escala para sua medida, foram introduzidos pelo sociólogo americano Emory S. Bogardus (1882-1973). Segundo Bogardus (1925), a distância social "refere-se aos graus e níveis de compreensão e sentimento que as pessoas experienciam em relação umas às outras. Explica a natureza de boa parte de sua interação. Registra o caráter das relações sociais" (s/p).

Sua proposta inicial era avaliar atitudes públicas de norteamericanos em relação a imigrantes de diferentes nacionalidades. Posteriormente, a escala foi modificada, associando rejeição social a atitudes estigmatizantes da população em torno de indivíduos com transtornos mentais (Angermeyer \& Matschinger, 1997; Coker, 2005).

De acordo com Blascovich, Mendes, Hunter e Lickel (2003), o desejo de distanciamento muitas vezes acontece a partir da inferência de uma ameaça, ocasionada por percepções distorcidas e compartilhadas das diferenças entre grupos, tais como: características físicas da pessoa rotulada, violações de regras sociais, falta de controle ou imprevisibilidade da doença e responsabilidade atribuída à pessoa pelo surgimento ou solução de sua condição de saúde. Tais distorções podem envolver atribuição de periculosidade e ameaça à saúde física do percebedor, despertando reações emocionais negativas como o medo, relativo mesmo a quem não é, de fato, perigoso.

Um estudo realizado por Peluso e Blay (2008) buscou avaliar a percepção da população em relação ao dependente de álcool na cidade de São Paulo e mostrou que esses indivíduos são percebidos como violentos e capazes de despertar reações negativas entre os membros da comunidade, como evitação e distanciamento. Outros estudos apontam que indivíduos dependentes de álcool e outras drogas são vistos como mais responsáveis por seu problema, mais violentos e mais imprevisíveis que indivíduos afetados por outros transtornos mentais (Angermeyer \& Matschinger, 1997; Link \& Phelan, 1999; Pescosolido, Monahan, Link, Stueve, \& Kikuzawa, 1999). Tais percepções condizem com o fato do consumo de álcool e outras drogas ser considerado como uma das principais condições estigmatizadas em todo o mundo (Corrigan et al., 2005; Fortney et al., 2004; Palm, 2006; Room, 2006).

Partindo da premissa de que muitos usuários de álcool e outras drogas frequentam variados tipos de serviços de saúde, o que promove o contato direto entre profissional e paciente, e que profissionais de saúde deveriam passar por uma formação teórica e prática sobre diversas condições de saúde, dentre elas, a dependência de substâncias psicoativas, pressupõe-se que tais fatores determinariam uma percepção diferenciada destes profissionais em relação à população a respeito do uso e/ou do usuário de substâncias. No entanto, estudos confirmam a hipótese de que o uso de álcool e outras drogas é um comportamento estigmatizado também pelos profissionais de saúde (Ronzani, Furtado, \& Higgins-Biddle, 2009; Silveira, Martins, \& Ronzani, 2009).

Profissionais de saúde que endossam atitudes autoritárias e estigmatizantes em relação a seus pacientes sem questionar os saberes e práticas nos quais se inserem (Rauter \& Peixoto, 2009) deixam de atender às reais demandas do usuário do serviço, promovendo tratamentos pobres e coercivos. Portanto, o estudo sobre o desejo de distância social dos profissionais de saúde em relação aos usuários de álcool e outras drogas pode contribuir para o manejo de estratégias adequadas de mudança de atitudes desses profissionais e uma melhora na qualidade do atendimento a esses pacientes (Ronzani, 2007; Room, 2006).

Desta forma, faz-se necessário investigar se há e quais são os fatores relacionados ao desejo de distanciamento social, para além dos processos de percepção social. Questiona-se se características do profissional de saúde como idade, gênero, estado civil e tempo de trabalho, podem ter influência no desejo de distância social, uma vez que não há na literatura nacional estudos que buscam avaliar tal relação como objetivo principal.

O presente estudo teve como objetivo avaliar o relacionamento entre variáveis sociodemográficas e o desejo de distanciamento social em relação aos dependentes de álcool, maconha e cocaína entre os profissionais de saúde da cidade de Juiz de Fora-MG, Brasil. A hipótese principal deste estudo foi de que não haveria relacionamento entre as variáveis sociodemográficas e o desejo de distância social, seja para o dependente de álcool, cocaína ou maconha, uma vez que, de acordo com a literatura, o desejo de distanciamento social em relação aos dependentes de substâncias psicoativas independe das características sociodemográficas de uma pessoa (Angermeyer \& Matschinger, 1997; Coker, 2005). Averiguou-se também a hipótese de que existiriam diferenças entre as percepções de distância social para a dependência de cada substância, assim como apontado no estudo de Link, Phelan, Bresnahan et al. (1999).

\section{Método}

\section{Participantes}

Participaram da pesquisa 183 profissionais de saúde de serviços especializados e ambulatoriais do município de Juiz de Fora, provenientes de serviços de Saúde Mental e de hospitais gerais. Optou-se pela escolha de hospitais de maior abrangência pela maior probabilidade de contato entre profissionais e pacientes com transtornos por uso de substâncias. A maioria dos profissionais de saúde era do gênero feminino $(67,8 \%)$, sendo $63,1 \%$ dos participantes, casados. Com relação à faixa etária, esta variou de 21 anos até 66 anos; a idade média foi de 44,1 anos $(D P=9,7$ anos $)$. O tempo de atuação dos profissionais de saúde nos serviços variou de 1 a 38 anos, sendo que 18,2 anos foi o tempo médio de profissão. No que diz respeito à profissão, $37,9 \%$ da amostra era constituída por auxiliares e técnicos de enfermagem, seguida por médicos $(26,4 \%)$, profissionais de 
nível superior $(18,1 \%)$, enfermeiros $(11,5 \%)$ e, por fim, outros técnicos e auxiliares $(6,0 \%)$. No que se refere ao convívio direto com usuários pesados ou dependentes de álcool e outras drogas no último ano, 67,4\% dos respondentes afirmaram que tinham contato seja pessoal ou profissional.

\section{Delineamento da pesquisa}

O presente estudo é parte de uma pesquisa mais ampla intitulada "Estereótipos e atribuição moral do uso de álcool entre profissionais de saúde de Juiz de Fora" ${ }^{1}$, desenvolvida pelo Pólo de Pesquisa em Psicologia Social e Saúde Coletiva da Universidade Federal de Juiz de Fora. Este estudo é do tipo exploratório, não controlado, de definição intencional da amostra. A definição dos serviços que compuseram a amostra foi feita seguindo um critério de conveniência que buscou uma maior homogeneidade entre os participantes. Optou-se por realizar um censo nos serviços de Saúde Mental, convidando todos a participar. Em relação aos hospitais gerais, constituiu-se uma amostra representativa dessas populações através da seleção aleatória estratificada de cada um dos universos.

\section{Procedimentos}

A coleta de dados nos serviços de Saúde Mental deu-se nos meses de outubro e novembro de 2008 e, nos hospitais gerais, aconteceu entre os meses de maio e agosto de 2009. Antes da aplicação na rede pública, foi conduzido um estudo piloto com profissionais de saúde para correção de eventuais problemas com os instrumentos. Após um agendamento prévio, os serviços de Saúde Mental do município e hospitais gerais foram visitados. Feita a apresentação da pesquisa e esclarecimento acerca dos aspectos éticos envolvidos, os profissionais eram convidados a ler e assinar o Termo de Consentimento Livre e Esclarecido (TCLE) para participar do estudo.

\section{Instrumentos}

Foram utilizados questionários autoaplicáveis, administrados em grupo ou individualmente, durante o horário de trabalho. Buscou-se intervir o mínimo possível na rotina de trabalho dos profissionais e manteve-se o controle de padronização da aplicação. Os instrumentos são apresentados abaixo:

Questionário Sociodemográfico. Objetivou caracterizar os participantes da pesquisa através de dados sociodemográficos como gênero, idade, estado civil, profissão e tempo de trabalho. Outro aspecto avaliado foi se no último ano, o profissional de saúde teve convívio direto (pessoal ou profissional) com algum usuário pesado ou dependente de álcool e outras drogas. Para conduzir a análise entre as variáveis estado civil e profissão, esta foi recategorizada em "Profissionais de Nível Superior" e "Profissionais de Nível Técnico", e aquela em "com cônjuge" e sem "cônjuge".

Vinhetas. Foram construídas a partir do estudo realizado por Link, Phelan, Bresnahan et al. (1999). Descrevem quadros de dependência de três substâncias - álcool, maconha e cocaína - segundo os critérios do DSM-IV. A cada participante foram apresentadas as seguintes vinhetas:

a) Dependência de Álcool: "José tem 45 anos de idade e sempre usa bebidas alcoólicas. No entanto, nos últimos meses
José tem bebido muito mais do que habitualmente para atingir os mesmos efeitos que antes. Toda vez que ele tenta diminuir ou parar de beber, ele fica agitado, tem náuseas, tremores e suores frios, que são aliviados pela ingestão de mais álcool. Mesmo sabendo que o álcool é nocivo para a saúde, José não consegue parar de beber. Ele tem tido problemas em casa com sua esposa e filhos. Além disso, seu patrão tem notado que ele tem sido bastante ausente no trabalho e a sua produção está bem inferior ao normal".

b) Dependência de Cocaína: "Marcos cheirou cocaína, pela primeira vez, em uma festa há um ano na companhia de amigos. Durante os últimos meses ele tem cheirado grandes quantidades por vez e tem perdido peso. Marcos tem gastado grande parte de suas economias para comprar cocaína. Quando seus amigos tentam falar com ele sobre as mudanças que tem apresentado, ele fica nervoso e tem reações explosivas. Além disso, também tem tido problemas na família. Marcos já tentou parar de cheirar cocaína algumas vezes, mas não obteve sucesso. Todas as vezes que tentou parar, se sentiu muito cansado e teve dificuldades para dormir, esses efeitos só foram aliviados quando ele usou mais cocaína. Marcos perdeu seu emprego há um mês devido às faltas constantes".

c) Dependência de Maconha: "Fabiano tem 25 anos e começou a fumar maconha na adolescência. Nos últimos meses, ele tem consumido muito mais do que de costume. Fabiano tem se mostrado ansioso e tem apresentado problemas de memória, atenção e concentração, sendo motivo de preocupação para sua família. Às vezes, quando está sob o efeito da maconha, se sente perdido em relação ao tempo e ao espaço. Além disso, seu patrão tem notado que ele tem sido bastante ausente no trabalho, está desmotivado e desinteressado e a sua produção está bem inferior ao normal".

As vinhetas foram utilizadas para avaliar, através do conjunto de 5 itens da Escala de Distância Social, comportamentos de evitar o contato com pessoas em relação as quais se tem crenças ou emoções negativas, ou seja, o quão desejado é o distanciamento social destas pessoas. Para cada vinheta, os participantes foram convidados a responder o quanto concordavam com as assertivas numa escala do tipo Likert de 7 pontos, com pontuação total variável de 5 a 35 , relativas ao seguinte enunciado: "Numa escala de 1 a 7 , onde 1 corresponde a "discorda totalmente" e 7 a "concorda totalmente", o quanto você gostaria de:

(1) Ser vizinho dessa pessoa; (2) Começar uma conversa com esta pessoa; (3) Ser amigo desta pessoa; (4) Trabalhar com esta pessoa; (5) Ter alguém da família casado com esta pessoa". As assertivas foram adaptadas do estudo de Link, Phelan, Bresnahan et al. (1999). Na avaliação de fidedignidade da escala, procedeu-se o teste de fidedignidade de Alfa de Cronbach, e observou-se os seguintes valores na correlação entre os itens: álcool $(\alpha=0,81)$; cocaína $(\alpha=0,82)$; maconha $(\alpha=0,83)$. Utilizou-se a rotação Varimax para a análise fatorial dos itens das três escalas. Para a escala de "álcool", a existência de um único fator foi explicada por $56,84 \%$ da variância total da escala (sendo o método adequado aos dados com $K M O=$ 0,750 e com $p \leq 0,001$ no teste de esfericidade de Bartlett). A existência de um único fator para a escala de "cocaína" foi 
explicada por $58,19 \%$ da variância total da escala (sendo o método adequado aos dados com $K M O=0,746$ e com $p \leq 0,001$ no teste de esfericidade de Bartlett). E, para a análise fatorial dos itens da escala de "maconha", a existência de um único fator foi explicada por $59,34 \%$ da variância total da escala (sendo o método adequado aos dados com $K M O=0,784$ e $\operatorname{com} p \leq 0,001$ no teste de esfericidade de Bartlett).

\section{Aspectos éticos}

O projeto recebeu aprovação dos Comitês de Ética em Pesquisa do Hospital Universitário HU/CAS da Universidade Federal de Juiz de Fora (UFJF) através do processo no ${ }^{-0}$ 0015/09.

Cada participante recebeu o TCLE, informando o tipo de pesquisa, seus objetivos e esclarecendo que a participação seria voluntária, não prevendo qualquer ressarcimento e sem prejuízo de qualquer natureza para os que não desejassem ou pudessem participar. No termo também se estabeleceu um compromisso com a privacidade de cada participante e a utilização confidencial e sigilosa dos dados colhidos.

Todas as atividades propostas foram negociadas dentro dos horários de serviço dos profissionais. Os resultados que serão aqui divulgados, respeitaram os critérios relatados acima com honestidade científica.

\section{Análises dos dados}

Os dados foram primeiramente digitados no software estatístico SPSS Versão 15.0, com a finalidade de corrigir eventuais erros de digitação e garantir a qualidade dos dados. Posteriormente, os dados foram organizados e analisados estatisticamente. Inicialmente foram utilizadas técnicas descritivas para apresentar as frequências $(n)$, porcentagens $(\%)$, média $(M)$, desvio padrão $(D P)$, valores mínimos (Min) e máximos (Max). A partir de então, verificou-se através do teste estatístico de Kolmogorov-Smirnov a hipótese nula de não existência de diferença estatística entre a distribuição amostral das escalas utilizadas neste estudo com uma distribuição normal. A hipótese nula foi rejeitada para a escala de "maconha" (com $p<0,05)$. Apesar deste resultado, a análise gráfica da distribuição dos escores da escala para "maconha" indicou a existência de simetria da distribuição, portanto, prosseguiramse as análises inferenciais através de estatísticas paramétricas. Inicialmente, utilizou-se a Análise de Variância (ANOVA) para medidas repetidas, com o objetivo de testar a hipótese nula de não existência de diferença entre as percepções de distância social dentre as escalas para cada substância. Tal análise foi complementada pelo teste post hoc de Bonferroni para comparar as médias das escalas duas a duas.

O teste $t$ de Student para amostras independentes - com a análise do teste da homogeneidade das variâncias de Levene - foi utilizado para testar a hipótese nula da não existência de diferença estatisticamente significativa entre a média dos escores de cada escala para as variáveis sociodemográficas dicotômicas: Gênero (masculino x feminino), Estado Civil (com cônjuge x sem cônjuge), Convívio (sim x não) e Nível Profissional (superior $\mathrm{x}$ técnico). Utilizou-se a correlação bivariada de Pearson ( $r$ de Pearson) para a análise da existência de correlação das variáveis Idade e Tempo de Serviço com os escores de cada uma das escalas de distância social. Adotou-se para todas as análises inferenciais o intervalo de confiança de $95 \%(p<0,05)$.

\section{Resultados}

Os resultados serão apresentados abaixo em dois tópicos: percepções de distância social para cada substância, e, relacionamento entre as variáveis sociodemográficas e distância social.

\section{Percepções de distância social para cada substância}

Álcool. O escore médio de percepção de distância social para o dependente de álcool foi de 18,20 com desvio padrão 6,73 , valores mínimo de 5 e máximo de 35 . O item que teve maior contribuição para o escore total, com valores mínimo de 1 e máximo de 7, foi: "Ter alguém na família casado com esta pessoa" $(M=2,19 ; D P=1,57)$, seguido por "Ser vizinho dessa pessoa" $(M=3,08 ; D P=1,64)$. O item que menos contribuiu para o escore total foi "Começar uma conversa com esta pessoa" $(M=4,93 ; D P=1,87)$.

Maconha. O escore médio de percepção de distância social para o dependente de maconha foi de 17,89 com desvio padrão 6,61, valores mínimo de 5 e máximo de 35 . O item que teve maior contribuição para o escore total, com valores mínimo de 1 e máximo de 7, foi: "Ter alguém na família casado com esta pessoa" $(M=2,29 ; D P=1,43)$, seguido por "Ser vizinho dessa pessoa" $(M=3,28 ; D P=1,66)$. O item que menos contribuiu para o escore total foi "Começar uma conversa com esta pessoa" $(M=4,65 ; D P=1,85)$.

Cocaína. O escore médio de percepção de distância social para o dependente de cocaína foi de 16,55 com desvio padrão 6,64, valores mínimo de 5 e máximo de 35 . O item que teve maior contribuição para o escore total, com valores mínimo de 1 e máximo de 7, foi: "Ter alguém na família casado com esta pessoa" $(M=2,13 ; D P=1,52)$, seguido por "Ser vizinho dessa pessoa" $(M=2,77 ; D P=1,60)$. O item que menos contribuiu para o escore total foi "Começar uma conversa com esta pessoa" $(M=4,62 ; D P=1,89)$.

Os dados completos para percepção de distância social da amostra total em relação aos itens das vinhetas das três dependências de substâncias - álcool, cocaína e maconha - estão descritos na Tabela 1 .

Os resultados da ANOVA de medidas repetidas para análise da diferença entre os escores das escalas para cada dependência de substância evidenciam a existência de diferença estatisticamente significativa entre as vinhetas $\left(F_{(2,320)}=10,47\right.$; $p \leq 0,001)$. Contudo, não se encontrou diferença no teste post hoc de Bonferroni entre as médias dos escores das escalas para dependência de "Álcool" e "Maconha", resultado diferente do observado nas comparações entre as escalas para dependência de "Álcool" e "Cocaína" ( $p \leq 0,001)$ e entre "Maconha" e "Cocaína” ( $p<0,002)$.

\section{Relacionamento entre as variáveis sociodemográficas} e distância social

Gênero, Estado Civil e Idade. Observou-se que não houve 
Tabela 1

Comparação da Média e Desvio Padrão da Escala de Distância Social para dependentes de álcool, cocaína e maconha

\begin{tabular}{|c|c|c|c|c|}
\hline Proposições & Vinhetas & $n$ & $M$ & $D P$ \\
\hline \multirow{3}{*}{$\begin{array}{l}\text { Ter alguém na família } \\
\text { casado com essa pessoa }\end{array}$} & Álcool & 183 & 2,19 & 1,57 \\
\hline & Maconha & 182 & 2,29 & 1,49 \\
\hline & Cocaína & 183 & 2,13 & 1,52 \\
\hline \multirow{3}{*}{$\begin{array}{c}\text { Ser vizinho dessa } \\
\text { Pessoa }\end{array}$} & Álcool & 183 & 3,08 & 1,64 \\
\hline & Maconha & 182 & 3,28 & 1,66 \\
\hline & Cocaína & 183 & 2,77 & 1,60 \\
\hline \multirow{3}{*}{$\begin{array}{c}\text { Trabalhar com essa } \\
\text { Pessoa }\end{array}$} & Álcool & 181 & 3,62 & 1,97 \\
\hline & Maconha & 180 & 3,53 & 1,80 \\
\hline & Cocaína & 182 & 3,22 & 1,82 \\
\hline \multirow{3}{*}{ Ser amigo dessa pessoa } & Álcool & 181 & 4,34 & 1,85 \\
\hline & Maconha & 179 & 4,08 & 1,78 \\
\hline & Cocaína & 181 & 3,87 & 1,87 \\
\hline \multirow{3}{*}{$\begin{array}{l}\text { Começar uma conversa } \\
\text { com essa pessoa }\end{array}$} & Álcool & 182 & 4,93 & 1,87 \\
\hline & Maconha & 182 & 4,65 & 1,85 \\
\hline & Cocaína & 182 & 4,62 & 1,89 \\
\hline \multirow{3}{*}{ Escore total } & Álcool & 179 & 18,20 & 6,73 \\
\hline & Maconha & 179 & 17,89 & 6,61 \\
\hline & Cocaína & 180 & 16,55 & 6,64 \\
\hline
\end{tabular}

diferença estatisticamente significativa para a média dos escores das vinhetas, comparando-se a média dos homens e mulheres para dependentes de "Álcool" $(t(177)=1,55 ; p<0,15)$, "Cocaína" $(t(178)=0,23 ; p<0,83)$ e "Maconha" $(t(177)=0,04$; $p<0,97)$. Também não foi encontrada diferença estatisticamente significativa, para a média dos escores das vinhetas, comparandose a média de profissionais que vivem com os cônjuges e sem os cônjuges para dependentes de "Álcool” $(t(151)=0,60 ; p<0,68)$, "Cocaína" $(t(154)=0,53 ; p<0,65)$ e "Maconha" $(t(152)=0,45$; $p<0,70)$. Ao se analisar a correlação entre a idade em anos e o escore nas escalas, encontrou-se que a resposta às escalas não estava correlacionada com a idade, tanto para a dependência de "Álcool" ( $p<0,19)$, como para de "Cocaína" $(p<0,14)$ e "Maconha" $(p<0,09)$. As médias dos escores das variáveis gênero e estado civil são encontradas na Tabela 2.

Tempo de Serviço e Nivel Profissional. Em relação à variável tempo de serviço, não foi encontrada correlação com as respostas às escalas, dependente de "Álcool" $(p<0,43)$, "Cocaína" ( $p<0,15)$ e "Maconha" $(p<0,06)$. Para a variável nível profissional (superior $x$ técnico), identificou-se que não houve diferença estatisticamente significativa para a média dos escores das vinhetas, comparando-se a média de profissionais de nível técnico e profissionais de nível superior para as escalas de dependência de "Cocaína" $(t(177)=1,32 ; p<0,19)$ e "Maconha" $(t(176)=0,12 ; p<0,91)$. Porém, foi encontrada diferença estatisticamente significativa para a média dos escores das vinhetas, a partir da comparação da média de profissionais de nível técnico $(M=20,12 ; D P=6,91)$ e profissionais de nível superior $(M=16,68 ; D P=6,23)$ para a escala de dependência de "Álcool" $(t(176)=3,43 ; p<0,01)$. As médias dos escores da variável nível profissional são encontradas na Tabela 2 .

Contato com o dependente. Não foi observada diferença estatisticamente significativa para a média dos escores das vinhetas, comparando-se a média de ter convivido e não ter convivido com algum usuário pesado ou dependente de álcool e outras drogas: "Álcool" $(t(175)=0,29 ; p<0,78)$, "Cocaína" $(t(176)=0,93 ; p<0,38)$, "Maconha" $(t(175)=2,03 ; p<0,05)$. As médias dos escores da variável contato são encontradas na Tabela 2 .

\section{Discussão}

No presente estudo, encontraram-se resultados semelhantes aos da literatura, no que se refere às evidências de que os efeitos negativos do rótulo em relação ao uso de álcool e outras drogas influenciam diretamente no desejo de distância social (Angermeyer \& Matschinger, 1997, 2005; Coker, 2005; Link, Phelan, Bresnahan et al., 1999).

A hipótese de que existiria diferença entre as percepções de distância social para cada substância foi parcialmente corroborada neste estudo. De acordo com os resultados, o distanciamento social foi maior para o dependente de cocaína do que para dependentes de álcool ou maconha. No estudo de Link, Phelan, Bresnahan et al. (1999), em que também foram 
Tabela 2

Média e Desvio Padrão das variáveis sociodemográficas na comparação entre dependentes de álcool, cocaína e maconha a partir da Escala de Distância Social.

\begin{tabular}{|c|c|c|c|c|c|}
\hline \multirow{7}{*}{ Gênero } & Vinhetas & Gênero & $n$ & $M$ & $D P$ \\
\hline & \multirow[t]{2}{*}{ Álcool } & Feminino & 121 & 18,70 & 6,85 \\
\hline & & Masculino & 58 & 17,16 & 6,40 \\
\hline & \multirow{2}{*}{ Cocaína } & Feminino & 122 & 16,62 & 6,68 \\
\hline & & Masculino & 58 & 16,40 & 6,61 \\
\hline & \multirow[t]{2}{*}{ Maconha } & Feminino & 120 & 17,91 & 6,80 \\
\hline & & Masculino & 59 & 17,86 & 6,27 \\
\hline \multirow{7}{*}{ Estado civil } & Vinhetas & Estado Civil & $n$ & $M$ & $D P$ \\
\hline & \multirow[t]{2}{*}{ Álcool } & Solteiro(a) & 42 & 18,57 & 7,41 \\
\hline & & Casado(a) & 111 & 17,97 & 6,56 \\
\hline & \multirow[t]{2}{*}{ Cocaína } & Solteiro(a) & 44 & 15,98 & 7,40 \\
\hline & & Casado(a) & 112 & 16,51 & 6,34 \\
\hline & \multirow[t]{2}{*}{ Maconha } & Solteiro(a) & 44 & 17,89 & 7,00 \\
\hline & & Casado(a) & 110 & 17,44 & 6,48 \\
\hline \multirow{7}{*}{$\begin{array}{c}\text { Nível } \\
\text { profissional }\end{array}$} & Vinhetas & Nível Profissional & $n$ & $M$ & $D P$ \\
\hline & \multirow[t]{2}{*}{ Álcool* } & Superior & 101 & 16,68 & 6,23 \\
\hline & & Técnico & 77 & 20,12 & 6,92 \\
\hline & \multirow{2}{*}{ Cocaína } & Superior & 101 & 15,94 & 6,32 \\
\hline & & Técnico & 78 & 17,26 & 7,01 \\
\hline & \multirow[t]{2}{*}{ Maconha } & Superior & 101 & 17,92 & 6,29 \\
\hline & & Técnico & 77 & 17,81 & 7,08 \\
\hline \multirow{7}{*}{ Contato } & Vinhetas & Contato & $n$ & $M$ & $D P$ \\
\hline & \multirow[t]{2}{*}{ Álcool } & Não & 57 & 17,93 & 6,72 \\
\hline & & Sim & 120 & 18,23 & 6,76 \\
\hline & \multirow[t]{2}{*}{ Cocaína } & Não & 59 & 15,83 & 6,86 \\
\hline & & Sim & 119 & 16,76 & 6,49 \\
\hline & \multirow[t]{2}{*}{ Maconha } & Não & 58 & 16,47 & 6,56 \\
\hline & & Sim & 119 & 18,50 & 6,57 \\
\hline
\end{tabular}

$* p<0,001$

utilizadas vinhetas para avaliar a distância social em relação a diferentes condições de saúde, entre elas, a dependência de substâncias, a pessoa descrita como dependente de cocaína foi apontada como a mais provavelmente violenta, seguido por dependentes de álcool. Os respondentes relataram maior desejo de distância social para a pessoa descrita como dependente de cocaína, mostrando a forte conexão entre distância social e a percepção de possível violência.

Não foi encontrada diferença estatisticamente significativa entre os escores das escalas para dependentes de álcool e maconha. Essa ausência de diferença entre os escores leva a alguns questionamentos.

Em primeiro lugar, no que diz respeito às drogas em si, estas possuem diferentes estatutos de legalidade na sociedade brasileira, implicando diferentes concepções sociais relacionadas à criminalidade e periculosidade da obtenção, uso e dependência, o que problematiza a ausência de diferenças. Contudo, segundo Jungerman, Laranjeira e Bressan (2005), a maconha é a droga ilícita mais usada em todo o mundo, o que possibilita a interpretação da existência de uma maior proximidade da sociedade com essa droga e um maior grau de aceitação no que diz respeito ao seu uso/dependência.

Em segundo lugar, poder-se-ia discutir aspectos relacionados à percepção social das sintomatologias, pois, assim como o álcool, a maconha é uma droga depressora do sistema nervoso, enquanto a cocaína, dada a excitação característica de seu uso, é mais relacionada a práticas violentas, as quais despertam medo e evitação.

A hipótese de que não haveria relacionamento entre as variáveis sociodemográficas e o desejo de distância social, seja para álcool, cocaína ou maconha foi amplamente corroborada, com exceção do aspecto nível profissional em relação ao álcool. No estudo de Coker (2005), os julgamentos de distância social também foram independentes de qualquer característica sociodemográfica da amostra do estudo.

Especificamente em relação à variável gênero, a literatura aponta que não existe relacionamento entre esta e o desejo de distância social em relação a dependentes de substâncias (Angermeyer \& Matschinger, 1997; Coker, 2005).

Estudos demonstram a existência de relação entre o nível de contato com a condição de saúde estigmatizada e a distância social. Neste estudo não foi encontrada tal relação. De acordo com os resultados da investigação realizada por Angermeyer e Matschinger (2005), independente dos respondentes serem familiares ou não a condições de saúde estigmatizadas, a percepção de imprevisibilidade e periculosidade foi 
significativamente aumentada pela rotulação. Entre os respondentes sem experiência pessoal com transtorno mental, a percepção de imprevisibilidade teve um efeito ainda maior na distância social, seguida por periculosidade. No estudo realizado por Corrigan et al. (2005), ao contrário dos adultos, adolescentes que têm maior familiaridade com alcoolistas apresentam maior probabilidade de estigmatização e, assim, possuem maior desejo de distância social. Estes resultados estão em concordância com Pereira (2002), no que diz respeito à sua concepção de não ser o simples contato, por si só, o que determina o aumento ou decréscimo do distanciamento social, havendo uma ampla e complexa esfera de níveis e naturezas de contato que interagem com o distanciamento.

No que diz respeito à variável nível profissional, os profissionais de nível superior tiveram média de escore total menor do que os profissionais de nível técnico, mostrando maior desejo de distanciamento social apenas para a dependência de álcool. O resultado sugere que as informações recebidas durante a formação superior não são suficientes para uma mudança de atitudes dos profissionais. Segundo Sales e Dimenstein (2009), existe uma defasagem entre os conteúdos discutidos na formação superior e a vivência desses profissionais em sua prática cotidiana. Este resultado vai de encontro ao estudo de Corrigan e Watson (2007), o qual aponta que pessoas que completam mais anos de educação têm mais conhecimento/ experiência com desordens psiquiátricas e endossam menos atitudes estigmatizantes e, consequentemente, possuem menor desejo de distanciamento social.

Como limitações do estudo, destacam-se a generalização dos resultados para profissionais de saúde, por se tratar de um estudo representativo para um município específico. $\mathrm{O}$ fato de o estudo ter sido conduzido por autorrelato, sem a observação direta do relacionamento interpessoal entre os profissionais de saúde e os indivíduos descritos é uma limitação adicional. Devese notar que os dados da Escala de Distância Social referem-se a percepções relativas à dependência de substâncias, e não somente a seu uso, de forma que interpretações alternativas das questões podem produzir resultados diferentes. Indica-se a possibilidade de inserção de questões no instrumento que busquem aumentar sua confiabilidade, por meio de um melhor esclarecimento da compreensão do respondente. Indica-se também a necessidade de estudos que investiguem possíveis diferenças de níveis de distanciamento social em relação à dependência, uso pesado e uso esporádico de substâncias. Desejabilidade social e inferências de respostas de comportamentos intencionais podem ser consideradas como limitações da escala.

Vale ressaltar que não foi realizada revisão de literatura acerca das variáveis sociodemográficas não consideradas na discussão. Por fim, diante da importância do tema e das implicações na saúde dos indivíduos com condições de saúde rejeitadas socialmente, tornam-se relevantes e necessárias novas pesquisas no campo da percepção e distância social.

\section{Conclusão}

De maneira geral, os resultados demonstraram que a percepção social é menos regida pelas características particulares dos indivíduos do que pelas rotulações socialmente compartilhadas. Enxerga-se aí a necessidade de aproximação de estudos acerca dos processos envolvidos na estigmatização e do distanciamento social como fenômenos correlacionados e relevantes para diversos campos sociais, como a saúde.

Concorda-se que a maneira como alguns profissionais de saúde percebem seus pacientes e conduzem o tratamento dos mesmos é essencial para a qualidade e adesão ao tratamento e realização de atividades de prevenção, sendo as atitudes estigmatizantes, uma barreira para o tratamento (Palm, 2006; Room, 2005; Silveira, Martins, \& Ronzani, 2009; Uçok, 2008).

Por fim, acrescenta-se a necessidade de uma reorganização dos serviços de saúde. Neste contexto, a literatura aponta que, para a promoção de uma atuação profissional mais integral, é necessário também, o desenvolvimento de capacitações profissionais voltadas para a atuação frente aos distúrbios relacionados ao uso de substâncias, que levem em conta as reais necessidades dos pacientes (Fulton, 2001; Ronzani, 2007).

\section{Agradecimentos}

Agradecemos à Fundação de Amparo à Pesquisa do Estado de Minas Gerais - FAPEMIG - pela concessão das bolsas de iniciação científica e de mestrado e ao Conselho Nacional de Desenvolvimento Científico e Tecnológico - CNPq - pela concessão de uma bolsa de iniciação científica e a todos que auxiliaram e participaram das fases do projeto, especialmente Thiago Constâncio Ribeiro Pereira, Víviam Vargas de Barros e Vinícius de Paula Menezes.

\section{Referências}

Angermeyer, M. C., \& Matschinger, H. (1997). Social Distance towards the Mentally ill: results of representative surveys in the Federal Republic of Germany. Psychological Medicine, 27, 131-141.

Angermeyer, M. C., \& Matschinger, H. (2005). Labeling - stereotype discrimination: an investigation of the stigma process. Social Psychiatry Epidemiology, 40, 391-395.

Blascovich, J., Mendes, W. B., Hunter, S. B., \& Lickel, B. (2003). Stigma, Threat, and Social Interactions. In T. F. Heatherton, R. E. Kleck, M. R. Hebl, \& J. G. Hull (Orgs.), The social psychology of stigma (pp. 307-333). New York: The Guilford Press.

Bogardus, E. S. (1925). Measuring social distances. Journal of Applied Sociology, 9, 299-308.

Coker, E. M. (2005). Selfhood and social distance: toward a cultural understanding of psychiatric stigma in Egypt. Social Science \& Medicine, 61, 920-930.

Corrigan, P. W., \& Watson, A. C. (2007). The stigma of psychiatric disorders and the gender, ethnicity and education of the perceiver. Community Mental Health Journal, 43(5), 439-458.

Corrigan, P., Markowitz, F. E., Watson, A., Rowan, D., \& Kubiak, M. A. (2003). An attribution model of public discrimination towards persons with mental illness. Journal of Health and Social Behavior, 44, 162-179.

Corrigan, P., Lurie, B. D., Goldman, H. H., Slopen, N., Medasani, K., \& Phelan, S. (2005). How adolescents perceive the stigma of mental illness and alcohol abuse. Psychiatric Services, 56, 544-550.

Fortney, J., Mukherjee, S., Curran, G., Fortney, S., Han, X., \& Booth, B. M. (2004). Factors associated with perceived stigma for alcohol use and treatment among at-risk drinkers. Journal of Behavioral Heatlh Services \& Research, 31(4), 418-429. 
Fulton, R. (2001). The stigma of substance use and attitudes of professionals: a review of the literature. (Tese de Pós-Doutorado não-publicada). Universidade de Toronto, Toronto.

Jungerman, F. S., Laranjeira, R., \& Bressan, R. A. (2005). Maconha: qual a amplitude de seus prejuízos? Revista Brasileira de Psiquiatria, 27(1), 5-6.

Link, B. G., \& Phelan, J. C. (1999). Labeling and stigma. In C. S. Aneshensel \& J. C. Phelan (Orgs.), Handbook of the sociology of mental health (pp. 481494). New York: Academic/Plenum Publishers.

Link, B. G., \& Phelan, J. C. (2001). Conceptualizing stigma. Annual Review of Sociology, 27, 363-385.

Link, B. G., Phelan, J. C., Bresnahan, M., Stueve, A., \& Pescosolido, B. A. (1999). Public conceptions of mental illness: labels, causes, dangerousness, and social distance. American Journal of Public Health, 89(9), 1328-1333.

Link, B. G., Yang, L. H., Phelan, J. C., \& Collins, P. Y. (2004). Measuring mental illness stigma. Schizophrenia Bulletin, 30(3), 511-541.

Palm, J. (2006). Moral concerns: treatment staff and user perspectives on alcohol and drug problems. (Tese de Doutorado não-publicada). Universidade de Estocolmo, Estocolmo.

Peluso, E. T. P., \& Blay, S. L. (2008). Public perception of alcohol dependence. Revista Brasileira de Psiquiatria, 30(1), 19-24.

Pereira, M. E. (2002). Psicologia social dos estereótipos. São Paulo: Editora Pedagógica e Universitária.

Pescosolido, B. A., Monahan, J., Link, B. G., Stueve, A., \& Kikuzawa, S. (1999). The public's view of the competence, dangerousness, and need for legal coercion of persons with mental health problems. American Journal of Public Health, 89(9), 1339-1345.
Rauter, C., \& Peixoto, P. T. C. (2009). Psiquiatria, saúde mental e biopoder: vida, controle e modulação no contemporâneo. Psicologia em Estudo, 14(2), 267-275

Rodrigues, A., Assmar, E. M. L., \& Jablonski, B. (2005). Cognição Social. In A. Rodrigues, E. M. L. Assmar \& B. Jablonski (Orgs.), Psicologia Social (pp. 67-96). Petrópolis: Vozes.

Ronzani, T. M. (2007). A reforma curricular nos cursos de saúde: qual o papel das crenças? Revista Brasileira de Educação Médica, 31(1), 38-43.

Ronzani, T. M., Furtado, E. F., \& Higgins-Biddle, J. (2009). Stigmatization of alcohol and other drug users by primary care providers in Southeast Brazil. Social Science \& Medicine, 69, 1-5.

Room, R. (2005). Stigma, social inequality and alcohol and drug use. Drug and Alcohol Review, 24, 143-155.

Room, R. (2006). Taking account of cultural and societal influences on substance use diagnoses and criteria. Research Report, 101(1), 31-39.

Sales, A. L. L. F., \& Dimenstein, M. (2009). Psicólogos no processo de Reforma Psiquiátrica: práticas em desconstrução? Psicologia em Estudo, 14(2), 277-285

Silveira, P. S., Martins, L. F., \& Ronzani, T. M. (2009). Moralização sobre o uso de álcool entre agentes comunitários de saúde. Psicologia: Teoria e Prática, 11, 62-75.

Uçok, A. (2008). Other people stigmatize... but, what about us? Attitudes of mental health professionals towards patients with schizophrenia. In J. ArboledaFlórez \& N. Sartorius (Orgs.), Understanding the stigma of mental illness: theory and interventions (pp. 108-116). Chichester: John Wiley \& Sons.

Rhaisa Gontijo Soares é mestranda em Psicologia (Bolsista CAPES) na Universidade Federal de Juiz de Fora. Endereço para correspondência: Rua Orestes Fabiano Alves, 21/301, São Pedro, Juiz de Fora-MG. CEP: 36037-120.Telefone: (32)9133-0510.E-mail: rhaisags@yahoo.com.br Pollyanna Santos da Silveira, mestre em Psicologia pela Universidade Federal de Juiz de Fora, é doutoranda em Psicobiologia (Bolsista CAPES) pela Universidade Federal de São Paulo. E-mail: pollyannassilveira@ gmail.com

Leonardo Fernandes Martins é mestrando em Psicologia (Bolsista CAPES) pela Universidade Federal de Juiz de Fora. E-mail: leomartinsjf@gmail.com

Henrique Pinto Gomide é graduando em Psicologia pela Universidade Federal de Juiz de Fora. Bolsista de Iniciação Científica FAPEMIG. E-mail: henriquepgomide@gmail.com

Thais Medeiros Lopes é graduanda em Psicologia pela Universidade Federal de Juiz de Fora.

E-mail: thaismlopes@gmail.com

Telmo Mota Ronzani, doutor em Ciências da Saúde pela Universidade Federal de São Paulo (UNIFESP), pós-doutor pela Universidade de São Paulo (USP) e University of Connecticut Health Center (UCHC), é professor Adjunto da Universidade Federal de Juiz de Fora, Bolsista de Produtividade CNPq e pesquisador FAPEMIG. E-mail: telmo.ronzani@ufjf.edu.br 